\title{
ON THE APPROXIMATION ORDER FROM CERTAIN MULTIVARIATE SPLINE SPACES
}

\author{
WOLFGANG DAHMEN ${ }^{1}$ and CHARLES A. MICCHELLII $I^{2}$
}

(Received 19 January 1983; revised 8 August 1983)

\begin{abstract}
In this paper, we determine the optimal controlled approximation rates from certain bivariate splines on regular meshes.
\end{abstract}

\section{Introduction}

Our motivation for this paper arose from some recent interesting studies of bivariate spline spaces on the regular triangular mesh shown below, [2, 3]. In particular, the determination of the optimal approximation orders for these spaces (depending on their polynomial degree and the order of continuity) is an open problem.

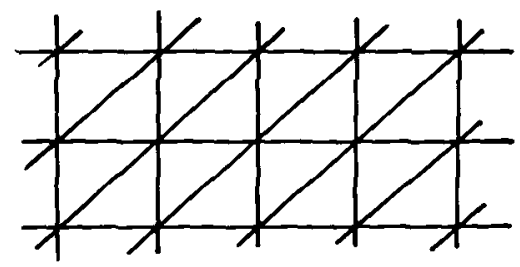

Apparently the difficulty in resolving this problem stems from the fact that the highest order for which all polynomials are locally contained in the spline spaces gives only an upper bound for the approximation order obtainable from the

\footnotetext{
${ }^{1}$ Fakultät für Mathematik, Universität Bielefeld, Universitätsstraße 1, 4800 Bielefeld, West Germany. ${ }^{2}$ IBM Thomas J. Watson Research Center, P.O. Box 218, Yorktown Heights, New York 10598, U.S.A (c) Copyright Australian Mathematical Society 1984, Serial-fee code 0334-2700/84
} 
corresponding splines. In fact, it is shown in [3] that $C^{1}$-cubics do locally contain all fourth order polynomials but the optimal approximation rate turns out to be only $O\left(h^{3}\right)$. On the other hand, it is also shown in [2] that the approximation properties of the full spline spaces are completely governed by those subspaces spanned by translates of certain so called 'box-splines' introduced in [1]. This reduces the problem to studying spaces of the type

$$
\mathscr{S}_{h}(\Phi)=\operatorname{span}\left\{\varphi_{i}(\dot{\dot{h}}-\alpha): i=1, \ldots, N, \alpha \in Z^{s}\right\}
$$

where $\Phi=\left\{\varphi_{1}, \ldots, \varphi_{N}\right\}$ is a collection of certain locally supported spline functions on $\mathbf{R}^{s}$ and in the case under consideration $s=2$. For a general collection $\Phi$ of functions, spaces of the type (1.1) have been intensely studied in the context of finite element analysis.

In this regard, two basic approaches should be mentioned. Using Fourier analysis, Fix and Strang $[5,6,8]$ developed quite a complete characterization of the approximation properties of $\mathscr{S}_{h}(\Phi)$ in an $L_{2}$-setting. An alternative approach based on Taylor expansion, was proposed by Mikhlin in [7] where approximation with respect to any $L_{p}$-norm was treated. His assumptions on $\Phi$ seem to be, however, more restrictive and not directly applicable to the spline spaces mentioned above.

This paper divides into two parts. In Section 2, we briefly revisit Fix and Strang's analysis to confirm the perhaps not surprising fact that their results can be extended to $L_{p}$-approximation for $1 \leqslant p \leqslant \infty$.

In particular, the exact controlled $L_{\infty}$-approximation order of $\mathscr{S}_{h}(\Phi)$ can as well be characterized in terms of the Fourier transforms $\hat{\varphi}_{i}, \varphi_{i} \in \Phi$. Since the Fourier transforms of box splines have a particularly simple structure we are able to apply these general results in Section 3 to the problems mentioned earlier. For instance, for $C^{2}$-quintics we obtain $O\left(h^{5}\right)$ as the optimal approximation rate.

\section{Controlled $L_{p}$-approximation}

Using standard multi-index notation, let

$$
\Pi_{k}=\left\{\sum_{|\beta| \leqslant k} c_{\beta} x^{\beta}: c_{\beta} \in \mathbf{R}\right\}
$$

denote the space of all polynomials of (total) degree $\leqslant k$. We write $\mathscr{S}(\Phi)$ instead of $\mathscr{S}_{1}(\Phi)$ and $\mathscr{S}(\varphi)$ if $\Phi=\{\varphi\}$.

Denoting by $\|\cdot\|_{p}(\Omega)$, the usual $L_{p}$-norm on $\Omega \subset \mathbf{R}^{s}, 1 \leqslant p \leqslant \infty$, we set $W_{p}^{m}(\Omega)=\left\{f:|f|_{p, m}(\Omega)=\left(\Sigma_{|\beta|=m}\left\|D^{\beta} f\right\|_{p}^{p}\right)^{1 / p}<\infty\right\}$. For $\Omega \subset \mathbf{R}^{s}$ we define

$$
\Omega_{h}=\left\{x \in \mathbf{R}^{s}: \operatorname{dist}(x, \Omega) \leqslant h\right\} .
$$


Adopting the terminology of [8] we say $\mathscr{S}(\Phi)$ admits "controlled $L_{p}$-approximation of order $k$ " or briefly

$$
\mathscr{S}(\Phi) \in A_{p, k}
$$

iff for any $f \in C^{\infty}\left(\mathbf{R}^{s}\right) \cap W_{p}^{k}\left(\mathbf{R}^{s}\right)$ there exist weights $\omega_{j, \alpha}^{h}$ such that for any closed domain $\Omega \subset \mathbf{R}^{s}$ the inequalities

$$
\left\|f-\sum_{j=1}^{N} \sum_{\alpha \in Z^{s}} \omega_{j, \alpha}^{h}\left(h^{-s / p} \varphi_{j}\left(\frac{\dot{h}}{h}-\alpha\right)\right)\right\|_{p}(\Omega) \leqslant C h^{k}|f|_{p, k}\left(\Omega_{r h}\right),
$$

and

$$
\begin{aligned}
\left\|\left\{\omega_{j, \alpha}^{h}\right\}\right\|_{l_{p}}(\Omega):= & \left(\sum_{j, \alpha}\left|\omega_{j, \alpha}^{h}\right|^{p}\right)^{1 / p} \leqslant C\|f\|_{p}\left(\Omega_{r h}\right), \\
& \operatorname{supp}\left(\varphi_{j}(\dot{\dot{h}}-\alpha)\right) \cap \Omega \neq \varnothing
\end{aligned}
$$

hold for constants $C, r$ independent of $h, \Omega, f$.

Note that (2.2) follows from (2.1) if the set $\left\{\varphi_{j}(\cdot-\alpha): j=1, \ldots, N, \alpha \in Z^{s}\right\}$ is linearly independent.

We begin by showing that for controlled approximation the choice of a particular norm does not matter in the following sense.

Proposition 2.1. $\mathscr{S}(\Phi) \in A_{\infty, k}$ implies $\mathscr{S}(\Phi) \in A_{p, k}$ for $1 \leqslant p \leqslant \infty$.

Proof. Following [7, page 50], we choose a $\Gamma \in C^{\infty}\left(\mathbf{R}^{s}\right)$ such that $\operatorname{supp}(\Gamma) \subset B$ $=\left\{x \in \mathbf{R}^{s},\|x\| \leqslant 1\right\}$ and

$$
\int_{\mathbf{R}^{s}} x^{\alpha} \Gamma(x) d x=\delta_{0, \alpha}, \quad 0 \leqslant|\alpha| \leqslant k
$$

Defining

$$
\tau_{h}(x)=h^{-s} \Gamma(x / h)
$$

and

$$
f_{h}(x)=\int_{\mathbf{R}^{s}} f(y) \tau_{h}(x-y) d y,
$$

we recall from [7, page 50] that for $f \in L_{p}\left(\mathbf{R}^{s}\right)$

$$
\left\|f_{h}\right\|_{p}(\Omega) \leqslant C\|f\|_{p}\left(\Omega_{h}\right)
$$

and, when $f \in W_{p}^{k}\left(\mathbf{R}^{s}\right)$,

$$
\left|f-f_{h}\right|_{p, l}(\Omega) \leqslant C|f|_{p, k}(\Omega) h^{k-l}, \quad 0 \leqslant l \leqslant k,
$$

where in both cases $C$ is independent of $\Omega$ and $h$. 
Now, for $f_{h}$ given by (2.3) there exist weights $u_{j, \alpha}^{h}$ such that

$$
S_{h}(x)=\sum_{j, \alpha} u_{j, \alpha}^{h} \varphi_{j}\left(\frac{x}{h}-\alpha\right)
$$

satisfies for some constant $r_{1}$,

$$
\left\|f_{h}-S_{h}\right\|_{\infty}(\Omega) \leqslant C_{1} h^{k}\left|f_{h}\right|_{\infty, k}\left(\Omega_{r_{1} h}\right) .
$$

In order to show that $\omega_{j, \alpha}^{h}:=h^{s / p} u_{j, \alpha}^{h}$ are appropriate weights for controlled $L_{p}$-approximation of $f$ we set

$$
G_{h, \alpha}=h\left(\bigcup_{j=1}^{N} \operatorname{supp}\left(\varphi_{j}\right)\right)+h \alpha, \quad \alpha \in Z^{s} .
$$

First note that integration by parts and Hölder's inequality yield

$$
\left|D^{\beta} f_{h}(x)\right|^{p} \leqslant C^{p} h^{-s} \int_{\|x-y\| \leqslant h}\left|D^{\beta} f(y)\right|^{p} d y, \quad|\beta| \leqslant k .
$$

Combining (2.6) and (2.7), we obtain for $x \in G_{h, \mathbf{a}}$

$$
\left|f_{h}(x)-S_{h}(x)\right|^{p} \leqslant C h^{p k-s}|f|_{p, k}^{p}\left(\left(G_{h, \alpha}\right)_{\left(1+r_{1}\right) h}\right) .
$$

Thus integrating (2.8) over $G_{h, \alpha}$ and using (2.5) gives

$$
\left\|f-S_{h}\right\|_{p}\left(G_{h, \alpha}\right) \leqslant C h^{k}|f|_{p, k}\left(G_{h, \alpha}\right)_{\left(1+r_{1}\right) h}
$$

which upon summation over $\alpha$ yields (2.1). Concerning (2.2) note that we have by hypothesis for some $0<r<\infty$,

$$
\begin{aligned}
\left\|\left\{\omega_{j, \alpha}^{h}\right\}\right\| P_{p}(\Omega) & \leqslant C_{1} \sum_{G_{h, \alpha} \cap \Omega \neq \varnothing} h^{s}\left(\left\|f_{h}\right\|_{\infty}\left(G_{h, \alpha}\right)_{r h}\right)^{p} \\
& \leqslant C_{2} \sum_{G_{h, \alpha} \cap \Omega \neq \varnothing}\|f\|_{p}^{p}\left(G_{h, \alpha}\right)_{(r+1) h} \leqslant C_{3}\|f\|_{p}^{p}\left(\Omega_{(r+1) h}\right)
\end{aligned}
$$

where we have used (2.7) in the second inequality.

We are now in a position to characterize those spaces belonging to $A_{p, k+1}$, $1 \leqslant p \leqslant \infty$.

THEOREM 2.1. The following are equivalent:

(i) For $0 \leqslant|\beta| \leqslant k$ there exist linear combinations $\psi_{\beta}$ of the $\varphi_{i}$ 's in $\Phi$ such that

$$
\begin{gathered}
\hat{\psi}_{0}(2 \pi \alpha)=\delta_{0 \alpha}, \quad \alpha \in Z^{s}, \\
\sum_{\gamma \leqslant \beta} \frac{D^{\gamma} \hat{\psi}_{\beta-\gamma}(2 \pi \alpha)}{\gamma ! i|\gamma|}=0, \quad \alpha \in Z^{s}-\{0\}, 1 \leqslant|\beta| \leqslant k .
\end{gathered}
$$

(ii) For any normed linear function space $(\mathscr{F},\|\cdot\|)$ on $\mathbf{R}^{s}$ for which translation is an isometry and restriction to closed subsets of $\mathbf{R}^{s}$ is a contraction one has: there exist 
bounded linear functionals $\mu_{i}, i=1, \ldots, N$, such that the map

$$
\left(Q_{h} f\right)(x)=\sum_{j=1}^{N} \sum_{\alpha \in Z^{s}} \mu_{j}(f(h(\cdot+\alpha))) \varphi_{j}\left(\frac{x}{h}-\alpha\right)
$$

satisfies

$$
\left|\mu_{j}(f(h(\cdot+\alpha)))\right| \leqslant c\left\|\left.f(h(\cdot+\alpha))\right|_{[-1,1]\}}\right\|
$$

and

$$
\left|f(x)-\left(Q_{h} f\right)(x)\right| \leqslant c \inf _{P \in \Pi_{k}}\left\|\left.(f-P)(h(\cdot+x))\right|_{[-r, r]^{*}}\right\|
$$

for some fixed constants $c<\infty, r>0$ and almost all $x \in \mathbf{R}^{s}$.

(iii) $\mathscr{S}(\Phi) \in A_{p, k+1}$, for all $p, 1 \leqslant p \leqslant \infty$.

$$
\text { (iv) } \mathscr{S}(\Phi) \in A_{\infty, k+1} \text {. }
$$

To prove Theorem 2.1 we observe first that (i) and (ii) of Theorem 2.1 are equivalent when $N=1$, i.e. $\Phi=\{\varphi\}$.

LEMMA 2.1. Condition (ii) in Theorem 2.1 holds for $\Phi=\{\varphi\}$ iff

$$
\hat{\varphi}(0) \neq 0, \quad D^{\beta} \hat{\varphi}(2 \pi \alpha)=0, \quad \alpha \in Z^{s}-\{0\},|\beta| \leqslant k .
$$

Proof. Condition (ii) of Theorem 2.1 implies $\mathscr{S}(\varphi) \in A_{2, k+1}$. Then (2.14) follows by [6, Theorem I]. The converse is shown in [4].

To prove Theorem 2.1 for $N \geqslant 1$ we closely follow the lines of $[6,7,8]$ where however (2.11) is required to hold also for $\alpha=0$. In order to show that it is sufficient to work with this weaker condition we again use a technique of [6] and define for $|\beta| \leqslant k, \quad 1=(1, \ldots, 1), \quad t_{\beta}(x):=(x-1)^{\beta}$ and $T_{\beta}(z)=$ $t_{\beta}\left(\exp \left(i z_{1}\right), \ldots, \exp \left(i z_{s}\right)\right)$ so that

$$
D^{\gamma} T_{\beta}(0)= \begin{cases}\beta ! i^{|\beta|}, & \gamma=\beta, \\ 0, & \gamma \neq \beta\end{cases}
$$

Hence for

$$
\hat{\Theta}=\sum_{|\beta| \leqslant k} T_{\beta} \hat{\psi}_{\beta}
$$

we obtain

$$
\Theta(x)=\sum_{|\beta| \leqslant k} \sum_{\gamma \leqslant \beta}\left(\begin{array}{l}
\beta \\
\gamma
\end{array}\right) \psi_{\beta}(x+\gamma) .
$$


Using the periodicity of $T_{\beta}(z)$ as well as (2.15) it is not hard to verify $\hat{\Theta}(2 \pi \alpha)=\hat{\psi}_{0}(2 \pi \alpha)=\delta_{0 \alpha}, \quad D^{\beta} \hat{\Theta}(2 \pi \alpha)=i^{|\beta| \beta !} \sum_{\gamma \leqslant \beta} \frac{D^{\gamma} \hat{\psi}_{\beta-\gamma}(2 \pi \alpha)}{\gamma ! i^{|\gamma|}}, \quad|\beta| \leqslant k$. Hence when $\psi_{\beta}$ satisfy (2.10), (2.11) condition (2.14) holds for $\Theta$ and Lemma 2.1 provides a map

$$
\sum_{\alpha} \mu(f(h(\cdot+\alpha))) \Theta\left(\frac{x}{h}-\alpha\right)
$$

satisfying (2.13). Using (2.16) to express (2.17) in terms of the $\varphi_{i} \in \Phi$ readily shows that (i) implies (ii). Observe that, when specializing (ii) to $L_{p}$-norms, (2.13) means

$$
\left|\mu_{j}(f(h(\cdot+\alpha)))\right| \leqslant C h^{-s / p}\|f\|_{p}\left([-h, h]^{s}+\alpha h\right)
$$

which proves (iii) with $\omega_{j, \alpha}^{h}=h^{s / p} \mu_{j}(f(h(\cdot+\alpha)))$. (iv) is an immediate consequence of (iii). On the other hand, by Proposition 2.1, (iv) also implies (iii). Finally, it is shown in $[5,6]$ that the case $p=2$ in (iii) already implies (i), finishing the proof.

Remarks. (i) Controlled approximation turns out to be equivalent to the existence of a uniformly bounded sequence of local linear (quasi-interpolant-type) approximation schemes (2.12) which realize the optimal approximation order. These approximation schemes may be determined with the aid of the conditions on the Fourier transforms of the $\varphi_{i} \in \Phi$ which will be illustrated in the next section.

(ii) It is shown in $[5,6]$ that the conditions $(2.10),(2.11)$ are equivalent to the existence of linear combinations $\psi_{\beta}$ of the $\varphi_{i}$ 's such that

$$
\frac{x^{\beta}}{\beta !}=\sum_{\gamma \leqslant \beta} \sum_{\alpha \in Z^{5}} \alpha^{\gamma} \frac{\psi_{\beta-\gamma}(x-\alpha)}{\gamma !}, \quad|\beta| \leqslant k .
$$

Note that, in general, condition (2.18) is strictly stronger than requiring that

$$
\Pi_{k} \subseteq \mathscr{S}(\Phi) .
$$

In fact, the counterexample in [3] gives an instance of $\Phi$ where $\Pi_{3} \subseteq \mathscr{S}(\Phi)$ but $\mathscr{S}(\Phi) \notin A_{\infty, 4}$ so that, by Theorem 2.1, (2.18) cannot hold for $k=3$. Note, however, that in this case the elements of $\Phi$ are box splines and translates of several box splines are never linearly independent because already the translates of each box spline are known to span $\Pi_{0},[1,4]$. 
If, however, the functions $\varphi_{i}(\cdot-\alpha), i=1, \ldots, N, \alpha \in Z^{s}$, are linearly independent then (2.18) is equivalent to (2.19) so that linear independence seems to be the right condition to make sure that the simple rule

$$
\mathscr{S}(\Phi) \in A_{p, k+1} \quad \text { iff } \quad \Pi_{k} \subseteq \mathscr{S}(\Phi)
$$

holds.

In order to confirm that in the case of linear independence (2.19) implies (2.18) we employ an inductive argument similar to the one indicated in [6] for the case $N=1$. First, we show that (2.18) holds for $\beta=0$. Clearly, (2.18) implies that for some constants, $1=\sum_{\alpha, j} c_{j, \alpha}^{0} \varphi_{j}(x-\alpha)$. Thus, $1=\sum_{\alpha, j} c_{j, \alpha-\gamma}^{0} \varphi_{j}(x-\alpha)$, for each $\gamma \in Z^{s}$. By linear independence we conclude $c_{j, \alpha}^{0}=c_{j, 0}^{0}, \alpha \in Z^{s}$. Setting

$$
\psi_{0}(x)=\sum_{j=1}^{N} c_{j, 0}^{0} \varphi_{j}(x)
$$

verifies that (2.18) holds for $\beta=0$. Suppose we have found for $\beta<\nu,|\nu| \leqslant k$, functions $\psi_{\mu}=\sum_{j=1}^{N} c_{j}^{\mu} \varphi_{j}$ satisfying (2.18) for $\mu<\nu$. We advance the induction by finding a $\psi_{\nu}$ such that $(2.18)$ holds for $\beta=\nu$. Let $x^{\nu} / \nu !=\sum_{\alpha, j} c_{j, \alpha}^{\nu} \varphi_{j}(x-\alpha)$. Then for $\gamma \in Z^{s}$

$$
(x+\gamma)^{\nu} / \nu !=\sum_{\alpha, j} c_{j, \alpha+\gamma}^{\nu} \varphi_{j}(x-\alpha)
$$

while, on the other hand, expanding $(x+\gamma)^{\nu}$ and using our induction hypothesis yields

$$
(x+\gamma)^{\nu} / \nu !=\sum_{\alpha, j}\left(c_{j, \alpha}^{\nu}+\sum_{\beta<\nu} \frac{\gamma^{\nu-\beta}}{(\nu-\beta) !} \sum_{\eta \leqslant \beta} \frac{\alpha^{\eta}}{\eta !} c_{j}^{\beta-\eta}\right) \varphi_{j}(x-\alpha) .
$$

Equating coefficients and setting $\alpha=0$ gives the relations

$$
c_{j, \gamma}^{\nu}=c_{j, 0}^{\nu}+\sum_{0<\beta \leqslant \nu} \frac{\alpha^{\beta}}{\beta !} c_{j}^{\nu-\beta} .
$$

Defining $\psi_{\nu}(x)=\sum_{j=1}^{N} c_{j, 0}^{\nu} \varphi_{j}(x)$ we have

$$
\begin{aligned}
x^{\nu} / \nu ! & =\sum_{\alpha, j}\left(c_{j, 0}^{\nu}+\sum_{0<\beta \leqslant \nu} \frac{\gamma^{\beta}}{\beta !} c_{j}^{\nu-\beta}\right) \varphi_{j}(x-\alpha) \\
& =\sum_{\alpha} \psi_{\nu}(x-\alpha)+\sum_{0<\beta \leqslant \nu} \frac{\alpha^{\beta}}{\beta !} \psi_{\nu-\beta}(x-\alpha),
\end{aligned}
$$

which was to be shown. 


\section{Application to translates of box-splines}

Following [1] we define for a given set of vectors $X=\left\{x^{1}, \ldots, x^{n}\right\} \subset \mathbf{R}^{s}$ such that

$$
\langle X\rangle:=\operatorname{span}\{X\}=\mathbf{R}^{s}
$$

the box spline $P\left(x \mid x^{1}, \ldots, x^{n}\right)$ or briefly $P(x \mid X)$ by requiring that

$$
\int_{\mathbf{R}^{s}} f(x) P\left(x \mid x^{1}, \ldots, x^{n}\right) d x=\int_{[0,1]^{n}} f\left(t_{1} x^{1}+\cdots+t_{n} x^{n}\right) d t_{1} \cdots d t_{n}
$$

holds for all $f \in C\left(\mathbf{R}^{s}\right)$.

Denoting by $|X|$ the cardinality of $X$ (counting repetitions) we associate with any $X$ as above the number

$$
d=d(X)=\max \left\{m:\langle X \backslash Y\rangle=\mathbf{R}^{s} \text { for all } Y \subset X,|Y|=m\right\} .
$$

Using the abbreviation $\mathscr{S}(X)=\mathscr{S}(P(\cdot \mid X))$ it is known that (cf. $[1,4])$

$$
\begin{gathered}
\Pi_{d} \subset \mathscr{S}(X), \quad \Pi_{d+1} \not \subset \mathscr{S}(X), \\
\mathscr{S}(X) \subset C^{d-1}\left(\mathbf{R}^{s}\right),
\end{gathered}
$$

and that $\mathscr{S}(X)$ is a space of piecewise polynomials of total degree $\leqslant n-s$. Moreover the Fourier transform of $P(\cdot \mid X)$ is easily calculated from (3.1) as

$$
\left(P(\cdot \mid X) \hat{)}(x)=\prod_{j=1}^{n} \frac{1-e^{-i x \cdot x^{j}}}{i x \cdot x^{j}} .\right.
$$

Here we are mainly interested in the case $s=2$ and the special sets

$$
X_{r, q, t}=\{\underbrace{e^{1}, \ldots, e^{1}}_{r}, \underbrace{e^{2}, \ldots, e^{2}}_{q}, \underbrace{e, \ldots, e}_{t}\}
$$

where $e^{1}=(1,0), e^{2}=(0,1), e=(1,1)$. However, our method applies in greater generality. In particular, for a given $n$ we consider

$$
d_{n}=\max \left\{d\left(X_{r, q, t}\right): r+q+t=n\right\}
$$

and the collection of box-splines with maximal order of smoothness

$$
\Phi_{n}:=\left\{P\left(\cdot \mid X_{r, q, t}\right): r+q+t \leqslant n, d\left(X_{r, q, t}\right)=d_{n}\right\} .
$$

Suppose $\Delta$ is the regular triangulation of $\mathbf{R}^{2}$ generated by multi-integer translates of the lines $\left\{t e^{1}: t \in \mathbf{R}\right\},\left\{t e^{2}: t \in \mathbf{R}\right\}$ and $\{t e: t \in \mathbf{R}\}$. Let $\Pi_{k, h \Delta}^{t}$ denote the space of all $l$ times continuously differentiable piecewise polynomials of degree $k$ on the scaled triangulation $h \Delta$. So, in view of (3.4) and the definition of $d_{n}$, we have that

$$
\mathscr{S}_{h}\left(\Phi_{n}\right) \subseteq \Pi_{n-2, h \Delta}^{d_{n}-1}
$$


The exact approximation orders from $\mathscr{P}\left(\Phi_{n}\right)$ are given in

THEOREM 3.1. For $1 \leqslant p \leqslant \infty$, one has

$$
\mathscr{S}\left(\Phi_{n}\right) \in \begin{cases}A_{p, 2 m} \backslash A_{\infty, 2 m+1}, & n=3 m, \\ A_{p, 2 m+1} \backslash A_{\infty, 2 m+2}, & n=3 m+1, n=3 m+2 .\end{cases}
$$

REMarK. (i) It is shown in [4] that when $X \subset Z^{s}, \hat{P}(\cdot \mid X)$ satisfies (2.14) for $k=d(X)$, i.e.

$$
\left(P(\cdot \mid X) \hat{)}(0) \neq 0, \quad D^{\beta}\left(P(\cdot \mid X) \hat{)}(2 \pi \alpha)=0, \quad \alpha \in Z^{s}-\{0\},|\beta| \leqslant d(X) .\right.\right.
$$

Thus, recalling that $\mathscr{S}(\Phi) \in A_{p, k+1}$ implies $\Pi_{k} \subset \mathscr{S}(\Phi)$, Lemma 2.1 combined with (3.3) yields

$$
\mathscr{S}(X) \in A_{p, d(X)+1} \backslash A_{p, d(X)+2},
$$

which was proved first in [1] for $p=\infty$ by different arguments. Moreover, one can readily check that for $n=3 m+l, l=0,1,2$,

$$
d_{n}= \begin{cases}2 m-1, & l=0,1, \\ 2 m, & l=2,\end{cases}
$$

Theorem 3.1 says that only for $l=1$ the approximation order from $\mathscr{S}\left(\Phi_{n}\right)$ exceeds the order attained by each of the subspaces $\mathscr{S}(X), P(\cdot \mid X) \in \Phi_{n}$.

(ii) It was pointed out in [2] that the approximation power of the spaces $\Pi_{k, \Delta h}^{\prime}$ is already completely determined by the span of all box splines contained in $\Pi_{k, \Delta h}^{\prime}$. Thus, by definition of $\mathscr{S}\left(\Phi_{n}\right)$, Theorem 3.1 also provides the exact approximation orders for the spaces $\Pi_{n-2, n \Delta}$. Note that $l=d_{n}-1$ is the highest order of continuity for which any given continuous function, say, can be approximated by elements of $\Pi_{n-2, h \Delta}^{\prime}$ arbitrarily well; (cf. [2]). Aside from the low order cases $n \leqslant 5$ and the case $n=3 m$ where $\left|\Phi_{n}\right|=1$, only nonmatching upper and lower bounds for these approximation orders were known, $[2,3]$.

Proof of Theorem 3.1. Let $n=3 m+l, l=0,2,1$.

$l=0$ : In this case, we have $\Phi_{n}=\left\{P\left(\cdot \mid X_{m, m, m}\right)\right\}$ so that the assertion readily follows from (3.7), (3.8).

$l=2$ : It is easy to see that

$\Phi_{n}=\left\{\varphi_{1}=P\left(\cdot \mid X_{m, m+1, m+1}\right), \varphi_{2}=P\left(\cdot \mid X_{m+1, m, m+1}\right), \varphi_{3}=P\left(\cdot \mid X_{m+1, m+1, m}\right)\right\}$.

Again (3.7), (3.8) affirm that

$$
\mathscr{S}\left(\Phi_{n}\right) \in A_{p, d_{n}+1}=A_{p, 2 m+1} .
$$


Suppose now that we even have $\mathscr{S}\left(\Phi_{n}\right) \in A_{p, 2 m+2}$. In view of (3.6) and (3.8) Theorem 2.1(i) would imply the existence of a linear combination

$$
\psi=a \varphi_{1}+b \varphi_{2}+c \varphi_{3}
$$

such that

$$
\hat{\psi}(0)=1, \quad D^{\beta} \hat{\psi}(2 \pi \alpha)=0, \quad|\beta|=d_{n}+1=2 m+1, \alpha \in Z^{2}-\{0\} .
$$

Note that (3.5) reduces to

$$
\left(P\left(\cdot \mid X_{r, s, t}\right) \hat{)}(x, y)=\rho(x)^{r} \rho(y)^{s} \rho(x+y)^{t}\right.
$$

where $\rho(x)=\left(1-e^{-i x}\right) /(i x)$. We must therefore have for $j \in Z-\{0\}$

$$
\begin{gathered}
0=\left.\left(\frac{\partial}{\partial x}\right)^{2 m+1} \hat{\psi}(x, y)\right|_{(2 \pi j, 0)}=(2 m+1) ! \rho^{\prime}(2 \pi j)^{2 m+1}(a+c), \\
0=\left.\left(\frac{\partial}{\partial y}\right)^{2 m+1} \hat{\psi}(x, y)\right|_{(0,2 \pi j)}=(2 m+1) ! \rho^{\prime}(2 \pi j)^{2 m+1}(b+c), \\
0=\left.\left(\frac{\partial}{\partial x}-\frac{\partial}{\partial y}\right)^{2 m+1} \hat{\psi}(x, y)\right|_{(2 \pi j,-2 \pi j)}=(2 m+1) ! \rho^{\prime}(2 \pi j)^{2 m+1}(a+b) .
\end{gathered}
$$

This homogeneous system clearly has only the trivial solution. This finishes the case $l=2$.

$l=1:$ For $n=3 m+1$ we have

$$
\begin{aligned}
\Phi_{n}=\left\{\varphi_{1}=P\left(\cdot \mid X_{m, m, m+1}\right), \varphi_{2}\right. & =P\left(\cdot \mid X_{m, m+1, m}\right), \varphi_{3}=P\left(\cdot \mid X_{m+1, m, m}\right), \\
\varphi_{4} & =P\left(\cdot \mid X_{m, m, m}\right), \varphi_{5}=P\left(\cdot \mid X_{m+1, m+1, m-1}\right), \\
\varphi_{6} & \left.=P\left(\cdot \mid X_{m+1, m-1, m+1}\right), \varphi_{7}=P\left(\cdot \mid X_{m-1, m+1, m+1}\right)\right\} .
\end{aligned}
$$

In view of (3.11) we need the following special case of Lemma 3.1 in [4],

$$
\begin{aligned}
D^{\beta}\left(\rho(x)^{r} \rho(y)^{s} \rho(x+y)^{l}\right)= & \sum_{\substack{\nu \in Z_{+}^{r}, \nu \in Z^{s}, \delta \in Z_{+}^{1} \\
|\nu|+|\nu|+|\delta|=|\beta| \\
(|\nu|,|\mu| \leqslant \beta}} \frac{\beta ! \mu ! \delta !\left(\beta_{1}-|\nu|\right) !\left(\beta_{2}-|\mu|\right) !}{\nu^{\prime}} \\
& \times \prod_{j=1}^{r} \rho^{\left(\nu_{j}\right)}(x) \prod_{j=1}^{s} \rho^{\left(\mu_{j}\right)}(y) \prod_{j=1}^{l} \rho^{\left(\delta_{j}\right)}(x+y)
\end{aligned}
$$

where

$$
D^{\beta}=\frac{\partial^{|\beta|}}{\partial x^{\beta_{1}} \partial y^{\beta_{2}}} .
$$


Showing that $\mathscr{S}\left(\Phi_{n}\right) \in A_{p, 2 m+1}$ reduces, by Theorem 2.1, to verifying (2.10), (2.11) which, in view of (3.6), (3.8) means that we have to find a linear combination

$$
\psi=\sum_{j=1}^{7} c_{j} \varphi_{j}
$$

such that

$$
\hat{\psi}(0,0)=1, \quad D^{\beta} \hat{\psi}(2 \pi \alpha)=0, \quad \alpha \in Z^{2}-\{0\},|\beta|=2 m .
$$

where $\varphi_{i}$ are defined in (3.12). Using (3.13) and $\rho(2 \pi j)=0, j \in Z-\{0\}$, it is easy to check that

$$
D^{\beta} \hat{\varphi}_{i}(2 \pi l, 2 \pi j)=0, \quad|\beta|=2 m, \quad l, j \in Z-\{0\}, l+j \neq 0 .
$$

Thus the only remaining homogeneous conditions to check in (3.14) arise for $(x, y) \in E=\{(0,2 \pi j),(2 \pi j, 0),(2 \pi j,-2 \pi j): j \in Z-\{0\}\}$. Straightforward use of (3.13) provides for $(0,2 \pi j), j \in Z-\{0\}$, the values

$$
D^{\beta} \hat{\varphi}_{1}(0,2 \pi j)=D^{\beta} \hat{\varphi}_{2}(0,2 \pi j)=D^{\beta} \hat{\varphi}_{7}(0,2 \pi j)=0, \quad|\beta|=2 m,
$$

$D^{\beta} \hat{\varphi}_{3}(0,2 \pi j)=D^{\beta} \hat{\varphi}_{4}(0,2 \pi j)$

$$
= \begin{cases}\frac{m !(m+k) !}{k !} \rho^{\prime}(2 \pi j)^{2 m}, & \beta=(m-k, m+k), k=0, \ldots, m, \\ 0, & \text { otherwise }\end{cases}
$$

$D^{\beta} \hat{\varphi}_{5}(0,2 \pi j)$

$$
= \begin{cases}\frac{(m-1) !(m+k) !}{(k-1) !} \rho^{\prime}(2 \pi j)^{2 m}, & \beta=(m-k, m+k), k=1, \ldots, m, \\ 0, & \text { otherwise, }\end{cases}
$$

$D^{\beta} \hat{\varphi}_{6}(0,2 \pi j)$

$$
= \begin{cases}\frac{(m+1) !(m+k) !}{(k+1) !} \rho^{\prime}(2 \pi j)^{2 m}, & \beta=(m-k, m+k), k=-1, \ldots, m, \\ 0, & \text { otherwise. }\end{cases}
$$

On the points $(2 \pi j, 0)$, we have

$$
D^{\beta} \hat{\varphi}_{1}(2 \pi j, 0)=D^{\beta} \hat{\varphi}_{3}(2 \pi j, 0)=D^{\beta} \hat{\varphi}_{6}(2 \pi j, 0)=0, \quad|\beta|=2 m,
$$

$D^{\beta} \hat{\varphi}_{2}(2 \pi j, 0)=D^{\beta} \hat{\varphi}_{4}(2 \pi j, 0)$

$$
= \begin{cases}\frac{m !(m+k) !}{k !} \rho^{\prime}(2 \pi j)^{2 m}, & \beta=(m+k, m-k), k=0, \ldots, m, \\ 0, & \text { otherwise }\end{cases}
$$


$D^{\beta} \hat{\varphi}_{5}(2 \pi j, 0)$

$$
= \begin{cases}\frac{(m-1) !(m+k) !}{(k-1) !} \rho^{\prime}(2 \pi j)^{2 m}, & \beta=(m+k, m-k), k=1, \ldots, m, \\ 0, & \text { otherwise, }\end{cases}
$$

$D^{\beta} \hat{\varphi}_{7}(2 \pi j, 0)$

$$
= \begin{cases}\frac{(m+1) !(m+k) !}{(k+1) !} \rho^{\prime}(2 \pi j)^{2 m}, & \beta=(m+k, m-k), k=-1, \ldots, m, \\ 0, & \text { otherwise. }\end{cases}
$$

Finally, on the remaining points $(2 \pi j,-2 \pi j)$ we have

$$
\begin{aligned}
& D^{\beta} \hat{\varphi}_{2}(2 \pi j,-2 \pi j)=D^{\beta} \hat{\varphi}_{3}(2 \pi j,-2 \pi j)=D^{\beta} \hat{\varphi}_{5}(2 \pi j,-2 \pi j)=0, \quad|\beta|=2 m, \\
& D^{\beta} \hat{\varphi}_{1}(2 \pi j,-2 \pi j)=D^{\beta} \hat{\varphi}_{4}(2 \pi j,-2 \pi j)= \begin{cases}(m !)^{2}(-1)^{m} \rho^{\prime}(2 \pi j)^{2 m}, & \beta=(m, m), \\
0, & \text { otherwise, }\end{cases} \\
& D^{\beta} \hat{\varphi}_{6}(2 \pi j,-2 \pi j) \\
& = \begin{cases}(-1)^{m-1}(m+1) !(m-1) ! \rho^{\prime}(2 \pi j)^{2 m}, & \beta=(m+1, m-1), \\
0, & \text { otherwise }\end{cases} \\
& D^{\beta} \hat{\varphi}_{7}(2 \pi j,-2 \pi j) \\
& = \begin{cases}(-1)^{m+1}(m-1) !(m+1) ! \rho^{\prime}(2 \pi j)^{2 m}, & \beta=(m-1, m+1), \\
0, & \text { otherwise. }\end{cases}
\end{aligned}
$$

With these conditions (3.14) reduces to the system of linear equations

$$
\begin{gathered}
c_{5}=c_{6}=c_{7}=0, \quad c_{1}+c_{4}=0, \quad c_{2}+c_{4}=0, \quad c_{3}+c_{4}=0, \\
c_{1}+c_{2}+c_{3}+c_{4}=1 .
\end{gathered}
$$

In fact, we obtain the equations $c_{1}+c_{4}=0, c_{6}=0$ and $c_{7}=0$ from the conditions (3.14) on the points $(2 \pi j,-2 \pi j)$ while $c_{3}+c_{4}=0$ and $c_{5}=0$ come from the points $(0,2 \pi j)$. The remaining homogeneous equations are derived from (3.14) on the points $(2 \pi j, 0)$. These equations have the unique solution $c_{1}=c_{2}=$ $c_{3}=-c_{4}=\frac{1}{2}$. Hence we conclude $\mathscr{S}\left(\Phi_{n}\right) \in A_{p, 2 m+1}$.

Next, we will show that $\mathscr{S}\left(\Phi_{n}\right) \notin A_{p, 2 m+2}$.

Suppose to the contrary that $\mathscr{S}\left(\Phi_{n}\right) \in A_{p, 2 m+2}$. Theorem 2.1 combined with (3.6) would guarantee the existence of linear combinations $\psi_{(0,0)}, \psi_{(1,0)}, \psi_{(0,1)}$ of the $\varphi_{i}$ 's in $\Phi_{n}$ such that for all $|\beta| \leqslant 2 m+1$

$$
\sum_{|\gamma| \geqslant 2 m} \frac{D^{\gamma} \hat{\psi}_{\beta-\gamma}(2 \pi \alpha)}{i^{|\gamma|} \gamma !}=0, \quad \alpha \in Z^{2}-\{0\}
$$

Specializing this relation for $|\beta|=2 m$ gives

$$
D^{\beta} \hat{\psi}_{(0,0)}(2 \pi \alpha)=0, \quad \alpha \in Z^{2}-\{0\}, \hat{\psi}_{(0,0)}(0,0)=1,
$$


while $\beta=(0,2 m+1)$ yields

$$
\frac{\left(\frac{\partial}{\partial y}\right)^{2 m} \hat{\psi}_{(0,1)}(0,2 \pi j)}{(2 m) !}+\frac{\left(\frac{\partial}{\partial y}\right)^{2 m+1} \hat{\psi}_{(0,0)}(0,2 \pi j)}{i(2 m+1) !}=0 .
$$

According to the above calculations, (3.15) determines $\psi_{(0,0)}$ uniquely as

$$
\psi_{(0,0)}=\frac{1}{2}\left(\varphi_{1}+\varphi_{2}+\varphi_{3}-\varphi_{4}\right) .
$$

Furthermore, it is easily checked that for all $j \in Z-\{0\}$

$$
\left(\frac{\partial}{\partial y}\right)^{2 m+1} \hat{\psi}_{(0,0)}(0,2 \pi j)=(2 m+1) ! \rho^{\prime}(2 \pi j)^{2 m+1} .
$$

On the other hand, our previous calculations show that the term $\left(\frac{\partial}{\partial y}\right)^{2 m} \hat{\psi}_{(0,1)}(0,2 \pi j)$ in (3.16) has the form $c \rho^{\prime}(2 \pi j)^{2 m}$ for some constant $c$. Hence for $\beta=(0,2 m+1)$, (3.16) would imply $c+(2 m+1) ! \rho^{\prime}(2 \pi j)=0$ for all $j \in Z$ $-\{0\}$, which is impossible. Thus there exists no solution of (3.15), (3.16), which completes the proof.

REMARK. Using $\psi_{(0,0)}$ defined above the construction in [4] provides an explicit quasi-interpolant for $\mathscr{S}\left(\Phi_{n}\right)$ which realizes the optimal approximation order.

Let us finally note that the above calculations also provide some immediate information about the case of nonoptimal smoothness.

To this end, let us write

$$
\hat{\psi}_{(0,0)}(x, y)=\hat{B}_{m}(x, y) \Gamma_{0}(x, y)
$$

where $B_{r}(x, y)=B\left(x, y \mid X_{r, r, r}\right)$ and $\Gamma_{0}(x, y)=\frac{1}{2}(\rho(x)+\rho(y)+\rho(x+y)-1)$. Consider for $k<m$ the linear combination $\psi_{k}(x, y)$ of box splines defined by its Fourier transform

$$
\hat{\psi}_{k}(x, y)=\hat{B}_{m-k}(x, y) \Gamma_{0}(x, y)^{3 k+1}
$$

where we assume that $n=3 m+l$. Defining

$$
\Phi_{n}^{\mu}=\left\{B\left(x, y \mid X_{r, q, t}\right): r+q+t \leqslant n=3 m+l, d\left(X_{r, q, t}\right)>\mu\right\},
$$

we clearly have $\mathscr{S}\left(\Phi_{n}\right) \subseteq \mathscr{S}\left(\Phi_{n}^{\mu}\right)$ for all $\mu \leqslant d_{n}-1$. Hence

$$
\psi_{k} \in \mathscr{S}\left(\Phi_{n}^{2(m-k)-2}\right) \subseteq \Pi_{n-2, \Delta}^{2(m-k)-2} .
$$

Since

$$
\begin{array}{ll}
D^{\beta} \hat{B}_{m-k}(2 \pi i, 2 \pi j)=0, & |\beta| \leqslant 3(m-k)-1,(i, j) \in Z^{2} \backslash E, \\
D^{\beta} \hat{B}_{m-k}(2 \pi i, 2 \pi j)=0, & |\beta| \leqslant 2(m-k)-1,(i, j) \in Z^{2} \backslash\{(0,0)\},
\end{array}
$$

and

$$
\left.D^{\beta}\left(\Gamma_{0}(x, y)^{3 k+l}\right)\right|_{(2 \pi i, 2 \pi j)}=0, \quad|\beta| \leqslant 3 k+l-1,(i, j) \in E,
$$


we conclude that

$$
D^{\beta} \hat{\psi}_{k}(2 \pi i, 2 \pi j)=0, \quad|\beta| \leqslant 2 m+k+l-1,(i, j) \in Z^{2} \backslash\{(0,0)\},
$$

provided that

$$
3(m-k)-1 \geqslant 2 m+k+l-1 \text {. }
$$

Hence, when

$$
m \geqslant 4 k+l
$$

we readily get

$$
\mathscr{S}\left(\Phi_{3 m+l}^{2(m-k)-2}\right) \in A_{p, 2 m+k+l}
$$

The case $k=0, l=1$, is covered by Theorem 3.1. The case $k=0, l=2$ shows that the approximation rate increases by one when the order of continuity decreases by one.

Note added in proof. After this paper was accepted for publication we became aware that there seems to exist no complete proof for the implication (iii) $\Rightarrow$ (i) in Theorem 2.1 which was stated by G. Fix and G. Strang in $[5,6,8]$. However, Fix and Strang did prove the equivalence of condition (i) in Theorem 2.1 and the existence of a quasi-interpolant (2.12), (2.17) which guarantees the optimality of the approximation rates in Theorem 3.1 for a correspondingly restricted notion of controlled approximation.

\section{References}

[1] C. de Boor and K. Höllig, "B-splines from parallelepipeds", J. Analyse Math. 42 (1983), 99-115.

[2] C. de Boor and K. Höllig, "Bivariate box splines and smooth pp. functions on a three direction mesh", J. Comput. Appl. Math. 9 (1983), 13-28.

[3] C. de Boor and K. Höllig, "Approximation order from bivariate $C^{1}$-cubics: A counterexample", Proc. Amer. Math. Soc. 87 (1983), 649-655.

[4] W. Dahmen and C. A. Micchelli, "Translates of multivariate splines", 52/53 (1983), 217-234. Linear Algebra Appl.

[5] G. Fix and G. Strang, "Fourier analysis of the finite element method in Ritz-Galerkin theory", Siud. Appl. Math. 48 (1969), 265-273.

[6] G. Fix and G. Strang, "A Fourier analysis of the finite element variational method", C.I. M. E.II, Ciclo Erice (1971), in Constructive aspects of functional analysis (ed. G. Geymonat), (Cremonese, Rome, 1973), 793-840.

[7] S. G. Mikhlin, Approximation on a rectangular grid (Sijthoff and Noordhoff, Alphen aan den Rijn, 1979).

[8] G. Strang, "The finite element method and approximation theory", in Numerical solution of partial differential equations, SYNSPADE 1970, Univ. of Maryland, College Park (ed. B. Hubbard), (Academic Press, New York, 1970). 